University of Wollongong

Research Online

Australian Institute for Innovative Materials -

Papers

Australian Institute for Innovative Materials

$1-1-2013$

Photodegradation in encapsulated silole-based polymer: Pcbm solar cells investigated using transient absorption spectroscopy and charge extraction measurements

Tracey M. Clarke

University of Wollongong, tclarke@uow.edu.au

Christoph Lungenschmied

Konarka Technologies

Jeff Peet

Konarka Technologies

Nicolas Drolet

Konarka Technologies

Kenji Sunahara

National Institute of Advanced Industrial Science and Technology

See next page for additional authors

Follow this and additional works at: https://ro.uow.edu.au/aiimpapers

Part of the Engineering Commons, and the Physical Sciences and Mathematics Commons

Research Online is the open access institutional repository for the University of Wollongong. For further information contact the UOW Library: research-pubs@uow.edu.au 


\title{
Photodegradation in encapsulated silole-based polymer: Pcbm solar cells investigated using transient absorption spectroscopy and charge extraction measurements
}

\begin{abstract}
Light induced degradation has been observed in the performance of organic solar cells in the absence of oxygen and a detailed analysis of the effect of this photodegradation on optical and electrical features has been accomplished. This photodegradation study has been performed on encapsulated photovoltaic blend devices comprised of the silole-based donor-acceptor polymer KP115 blended with [6,6]-phenyl C61-butyric acid methyl ester (PCBM). Photodegradation induces an almost $20 \%$ decrease in power conversion efficiency, primarily as a result of a reduction in short circuit current, JSC. The initial burn-in phase of the photodegradation has been examined using a combination of transient absorption spectroscopy and charge extraction measurements, including photo-CELIV (charge extraction by linearly increasing voltage) and time-resolved charge extraction using a nanosecond switch. These measurements reveal a bimodal KP115 polaron population, comprised of both delocalised and localised/ trapped charge carriers. The photodegradation results are consistent with an alteration of this bimodal KP115 polaron population, with the polarons becoming trapped in a broader, deeper density of localised states. Under laser illumination and at open circuit conditions, this enhanced trapping after light soaking inhibits charges from undergoing bimolecular recombination, leading to higher extracted charge densities at long times. At the lower charge densities operating at short circuit conditions and under continuous white light illumination, where bimolecular recombination is much less significant, the JSC decreases after light soaking due to a reduction in the efficiency of trapped charge carrier extraction.

Disciplines

Engineering | Physical Sciences and Mathematics

\section{Publication Details}

Clarke, T. M., Lungenschmied, C., Peet, J., Drolet, N., Sunahara, K., Furube, A. \& Mozer, A. J. (2013).

Photodegradation in encapsulated silole-based polymer: Pcbm solar cells investigated using transient absorption spectroscopy and charge extraction measurements. Advanced Energy Materials, 3 (11), 1473-1483.

\section{Authors}

Tracey M. Clarke, Christoph Lungenschmied, Jeff Peet, Nicolas Drolet, Kenji Sunahara, Akihiro Furube, and Attila J. Mozer
\end{abstract}


DOI: 10.1002/aenm.201300337

Article type: Full Paper

\section{Photodegradation in encapsulated silole-based polymer:PCBM solar cells investigated using transient absorption spectroscopy and charge extraction measurements}

Tracey M. Clarke, * Christoph Lungenschmied, Jeff Peet, Nicolas Drolet, Kenji Sunahara, Akihiro Furube, and Attila J. Mozer*

[*] Tracey M. Clarke, Attila J. Mozer

ARC Centre of Excellence for Electromaterials Science, Intelligent Polymer Research Institute, University of Wollongong, North Wollongong, NSW 2500, Australia E-mail: tclarke@uow.edu.au, attila@uow.edu.au

Christoph Lungenschmied, Jeff Peet, Nicolas Drolet

Konarka Technologies, 116 John St., Suite 12, Lowell, Massachusetts 01852, USA.

Kenji Sunahara, Akihiro Furube

National Institute of Advanced Industrial Science and Technology (AIST), Tsukuba Central 5, 1-1-1 Higashi, Tsukuba, Ibaraki 305-8565, Japan

Keywords: charge transport, conducting polymer, organic solar cell, photodegradation, transient absorption spectroscopy

Light induced degradation has been observed in the performance of organic solar cells in the absence of oxygen and a detailed analysis of the effect of this photodegradation on optical and electrical features has been accomplished. This photodegradation study has been performed on encapsulated photovoltaic blend devices comprised of the silole-based donor-acceptor polymer KP115 blended with [6,6]-phenyl C61-butyric acid methyl ester (PCBM). Photodegradation induces an almost $20 \%$ decrease in power conversion efficiency, primarily as a result of a reduction in short circuit current, $J_{S C}$. The initial burn-in phase of the photodegradation has been examined using a combination of transient absorption spectroscopy and charge extraction measurements, including photo-CELIV (charge extraction by linearly increasing voltage) and time-resolved charge extraction using a nanosecond switch. These measurements reveal a bimodal KP115 polaron population, comprised of both 
delocalised and localised/trapped charge carriers. The photodegradation results are consistent with an alteration of this bimodal KP115 polaron population, with the polarons becoming trapped in a broader, deeper density of localised states. Under laser illumination and at open circuit conditions, this enhanced trapping after light soakings inhibits charges from undergoing bimolecular recombination, leading to higher extracted charge densities at long times. At the lower charge densities operating at short circuit conditions and under continuous white light illumination, where bimolecular recombination is much less significant, the $J_{S C}$ decreases after light soaking due to a reduction in the efficiency of extraction of trapped charge carriers.

\section{Introduction}

Even though efficiency records of organic solar cells based on blends of conjugated polymers and fullerene derivatives have been frequently broken over recent years, insufficient device lifetime may threaten the widespread implementation of this versatile technology. The present certified world record for a single junction polymer solar cell has recently surpassed $9 \%{ }^{[1]}$ Given the trend in efficiencies, the commercial success of this promising technology is increasingly possible. However, with so much emphasis on increasing power conversion efficiencies, relatively little attention has been applied to the stability of such organic photovoltaic devices. ${ }^{[2,3]}$ This is a key issue and considerable research is required to investigate mechanisms of degradation and strategies to enhance device lifetime.

A recent work addressing this point examined the effect of long-term photodegradation on encapsulated PCDTBT:PC $70 \mathrm{BM}$ solar cells. ${ }^{[4]}$ This high-performing blend, which has achieved efficiencies of over $7 \%,,^{[5,6]}$ exhibited an initial sharp loss in performance upon photo-illumination over the first few hundred hours (the burn-in phase), followed by a 
remarkably stable efficiency over several thousand hours. The degradation mechanism was concluded to be a photochemical reaction in the active layer that creates sub-bandgap states, thereby increasing the energetic disorder of the system. Further work done by Leclerc et al., ${ }^{[7]}$ albeit under ambient conditions (air), showed that the photo-oxidation mechanism in this blend involved polymer chain scission and cross-linking reactions. The burn-in phase has been noted in other blends, such as P3HT:PCBM, ${ }^{[8]}$ and may be a general phenomenon of these type of organic solar cells.

A polymer that is beginning to receive considerable attention is KP115, poly [(4,4'- bis (2ethylhexyl) dithieno [3,2-b:2',3'-d] silole) -2,6-diyl-alt-(2,5-bis 3-tetradecylthiophen-2-yl thiazolo 5,4-d thiazole)-2,5diyl]. ${ }^{[9-13]}$ This polymer, although it does not attain the high efficiencies of PCDTBT:PCBM in thin $(<100 \mathrm{~nm})$ active layer thickness devices, has particularly interesting characteristics in thick (> $200 \mathrm{~nm}$ active layer) devices. KP115:PCBM devices, which can reach efficiencies of $5 \%$, possess the rare but highly desirable characteristic that the active layer can be considerably thicker without a detrimental effect on the efficiency. However, we also observed that photodegradation effects have a greater impact on the efficiency of photovoltaic devices with thicker active layers (vide infra). Since commercially produced polymer solar cells will likely have thicker active layers to maximise light absorption and to facilitate the high speed coating of consistent layer thicknesses, it is vital to examine the degradation mechanisms of photovoltaic devices that exhibit good performance using thicker active layers.

Here we present a detailed study of the photodegradation of $\mathrm{KP} 115: \mathrm{PC}_{60} \mathrm{BM}$ photovoltaic devices. The devices studied here have an inverted geometry, with a Ag/hole-injecting layer (HIL)/active layer (140 nm thickness)/ electron-injecting layer (EIL)/ITO structure, where the HIL and EIL are Konarka proprietary materials. Encapsulation with glass to inhibit oxygen 
and water degradation was employed; however, as previously noted by several research groups, photodegradation can still be a significant issue. ${ }^{[2,4]}$ Like PCDTBT:PC ${ }_{70} \mathrm{BM},{ }^{[4]}$ KP115:PCBM shows an initial burn-in phase in which the efficiency drops sharply over the first $\sim 100$ hours, after which a much slower decay in efficiency is observed. This is presented in Figure 1, where the structure of KP115 is shown in the inset. However, in this case the drop in efficiency results from a substantial decrease in $J_{S C}$ (rather than $V_{O C}$ and fill factor, as in PCDTBT:PCBM). The aim of this study is to understand the origin of this loss of device performance during the burn-in phase of photodegradation in such systems. We use a combination of time-resolved charge extraction and optical techniques to show that this drop in efficiency with photodegradation is a result of a decrease in charge extraction efficiency due to an increased contribution from localised sub-bandgap states.

In section 2.1. the detrimental consequences of light soaking on the photovoltaic performance of KP115 based devices is illustrated. Section 2.2. covers the changes in the optical features of the polymer:fullerene blend. While the steady-state absorption spectrum shows little alteration upon light soaking, transient absorption reveals changes in the features attributed to localised polymer polarons. Sections 2.3. to 2.6. deal with a variety of charge extraction methods. Time-resolved charge extraction using a nanosecond switch (TRCE, section 2.3.) reveals slower bimolecular recombination of trapped charge carriers in light soaked KP115:PCBM , while photovoltage decays (section 2.4.) provides further insight into the density of states distribution. Photo CELIV (Charge Extraction by Linearly Increasing Voltage, section 2.5.) allows for the determination of charge carrier mobility before and after light soaking, which remains virtually unchanged. However, it is revealed that more charges are extracted and the photocurrent becomes more dispersive. Finally, in Section 2.6., the results of the transient photocurrent decay measurements are presented. After light soaking, more current at longer times is detected, when predominantly trapped charges are extracted. 


\section{Results}

\subsection{Current density - Voltage $(J V)$ characteristics}

Current-voltage curves recorded under $100 \mathrm{~mW} \mathrm{~cm}^{-2}$ simulated AM 1.5 illumination and in the dark are shown in Figure 2 for the KP115:PCBM (1:2) device before and after 72 hour light soaking, while the measured photovoltaic parameters are displayed in Table 1. Comparing the curves reveals a change in the slope of the $J V$ curve in the third and fourth quadrant. After light soaking, less current is observed at comparable voltage. This discrepancy becomes increasingly pronounced as the voltage moves from $-2 \mathrm{~V}$ towards the maximum power point. The primary consequence of this behaviour is a significant decrease in the short circuit current, $J_{S C}$, which drops by $14 \%$. The open circuit voltage $V_{O C}$ does not change and the fill factor (FF) shows only a small decrease. The overall decrease in device performance from $4.4 \%$ to $3.6 \%(18 \%)$ therefore primarily results from the loss of $J_{S C}$. The focus of this paper is to determine the origin of this initial burn-in loss of device performance. The three contributors to $J_{S C}$ are the efficiencies of photon absorption, free charge carrier photogeneration and charge collection. Each of these possibilities will be addressed in the following sections.

Table 1. KP115:PCBM (1:2) device performance before and after a 72 hour light soak. 


\begin{tabular}{|l|l|l|}
\hline Parameter & Prior to light soaking & After light soaking \\
\hline$J_{S C}\left(\mathrm{~mA} \cdot \mathrm{cm}^{-2}\right)$ & 11.8 & 10.1 \\
\hline$V_{O C}(\mathrm{~V})$ & 0.64 & 0.64 \\
\hline FF & 0.59 & 0.57 \\
\hline Efficiency (\%) & 4.4 & 3.6 \\
\hline
\end{tabular}

\subsection{Steady state and transient absorption spectroscopy}

The ground state absorption spectrum of an encapsulated KP115:PCBM film before and after light soaking is shown in Figure 3. Very little change has occurred, with both spectra showing two bands at 590 and $640 \mathrm{~nm}$, with a weak shoulder at $545 \mathrm{~nm}$ and the broad PCBM absorption shoulder at $335 \mathrm{~nm}$ (see Supporting Information Figure S1). No peak shifting is observed upon light degradation across the entire spectrum, although a very small decrease in absorption of $<0.02$ is present for the lowest energy peak and at $\sim 335 \mathrm{~nm}$ (PCBM absorption). As such, a significant decrease in photon absorption cannot be the cause of the decreased $J_{S C}$. Furthermore, the lack of change in the absorption spectrum suggests that no significant concentration of photodegradation products has been formed that, for example, reduces the conjugation length of the polymer. ${ }^{[7]}$ It should be noted, however, that studies that observe such effects upon photodegradation tend to use accelerated ageing conditions rather than the 1 sun used here, or are performed under ambient conditions. ${ }^{[14]}$ Photodegradation products may indeed be present here but could be at too low a concentration to be observed using this technique. The small but reproducible decreases in absorbance observed for the lowest energy and PCBM peaks may also be the beginning of a slight reduction in crystallinity. ${ }^{[15,16]}$ 
Transient absorption spectroscopy (TAS) is a key technique as it directly monitors the optical absorption of photogenerated transient species. For polymer:PCBM blends, these are typically charge carriers or triplet states on the nanosecond-millisecond timescales employed here. TAS provides information on the identity, yield and recombination of these transient species. This method is widely-known and has previously been applied to numerous polymer:blend systems. ${ }^{[12,17-25]}$ In general, the charge carrier decay kinetics exhibit a power law decay $(\triangle O D$ $\left.\propto t^{\alpha}\right)$; this has been observed in P3HT, ${ }^{[26]}$ polyselenophenes, ${ }^{[27]}$ and MDMO-PPV, ${ }^{[28,29]}$ all blended with various fullerene derivatives. This power law behaviour is consistent with models describing bimolecular recombination of dissociated charge carriers in the presence of an exponential distribution of localised (trapped) states. ${ }^{[30]}$ The value of $\alpha$ (the gradient of the power law) provides an indication of the energetic distribution of polaron localised states, where a shallow gradient with a low $\alpha$ value suggests the presence of energetically deep localised states. Thermal activation of the charge carriers out of these states is required before recombination can occur; this is the rate-limiting step. Conversely, steeper gradients are correlated with shallower localised states such that an $\alpha$ of one denotes pure (trap-free) bimolecular recombination. An excitation density-dependent fast phase is often observed prior to the slow trap-limited power law phase, assigned to recombination of free charge carriers generated when the density of photogenerated polarons exceeds the density of localised states. At low light intensities (small charge carrier densities) only the localised states are filled and thus this fast phase is not observed. Instead, the charge carrier dynamics are dominated by relatively slow, trap-limited recombination.

The presence of these localised states is sometimes also evident from the transient absorption spectra. P3HT:PCBM has been studied the most in this respect, where its transient absorption spectrum generally exhibits two distinct peaks at approximately 700 and $1000 \mathrm{~nm} \cdot{ }^{[20,31-33]}$ The $700 \mathrm{~nm}$ band has typically been attributed to mobile, delocalised positive polarons in 
crystalline regions of the P3HT domains while the latter has been attributed to positive polarons localised (trapped) in disordered amorphous P3HT domains. This assignment is supported by the fact that these two bands often have different decay kinetics, with the delocalised polaron band decaying via pure bimolecular recombination while the localised polarons exhibit slower trap-limited bimolecular recombination. ${ }^{[20]}$ In the case of P3HT, the positive polaron has a substantially higher molar extinction coefficient compared to the negative PCBM anion, thus the latter's absorption at $1070 \mathrm{~nm}$ is not visible and the polymer polaron band dominates the spectrum.

The transient absorption spectrum of the control KP115:PCBM device (before light soaking) was measured on both the early nanosecond and microsecond timescales, shown in Figures 4a and $\mathbf{4 b}$ respectively. The early nanosecond timescale shows two very broad overlapping bands at approximately 750 and $1050 \mathrm{~nm}$. These bands cannot be assigned to the polymer singlet state as time-resolved photoluminescence data (to be published elsewhere) shows the polymer singlet state emission to be $99 \%$ quenched within 120 ps in a KP115:PCBM (1:2) film. It is evident from the early nanosecond data in Figure 4a that the two overlapping bands decay on different timescales, with the $750 \mathrm{~nm}$ band decaying much more quickly. At $1 \mathrm{~ns}$, the $750 \mathrm{~nm}$ band is twice the amplitude of the $1050 \mathrm{~nm}$ band, but after only $15 \mathrm{~ns}$, the two amplitudes are approximately equivalent. The effect of this difference in kinetics is evident on the microsecond timescale (Figure 4b). At $1 \mu \mathrm{s}$, the slowly decaying band, now centred at $1080 \mathrm{~nm}$, is much more prominent and clearly defined. The rapidly decaying band is still visible as a feature at wavelengths below $750 \mathrm{~nm}$. Most of the decay has already occurred for this band and only a small tail remains on the microsecond timescale.

One method to establish whether the transient absorption peak at $\sim 1080 \mathrm{~nm}$ in Figure $4 \mathrm{~b}$ can be attributed to polymer KP115 polarons or PCBM anions (which absorb nearby, at $1070 \mathrm{~nm}$ ) 
is to measure the transient absorption spectrum of $\mathrm{KP} 115: \mathrm{PC}_{70} \mathrm{BM}$. The $\mathrm{PC}_{70} \mathrm{BM}$ anion absorbs further to the red, at $1370 \mathrm{~nm},{ }^{[34]}$ and thus is unlikely to interfere with shorter wavelength polymer polaron bands. The microsecond timescale transient absorption spectrum of $\mathrm{KP} 115: \mathrm{PC}_{70} \mathrm{BM}$ is shown in Figure 4c, appearing very similar to the $\mathrm{KP} 115: \mathrm{PC}_{60} \mathrm{BM}$ spectrum in Figure 4b. The $1080 \mathrm{~nm}$ band remains, thus providing clear support for the assignment of this peak to KP115 polarons rather than the PCBM anion. Indeed, there is little evidence of the $\mathrm{PC}_{70} \mathrm{BM}$ anion peak, suggesting that the KP115 polaron has a substantially higher molar extinction coefficient and thus dominates the spectrum. It is also important to note that the feature at wavelengths below $750 \mathrm{~nm}$ is also still present, indicating that it too can be attributed to the KP115 polaron.

These results have a key similarity to results previously reported for $\mathrm{P} 3 \mathrm{HT}: \mathrm{PCBM},{ }^{[20]}$ in terms of a bimodal (localised and delocalised) polaron band behaviour. Such behaviour has only been observed in a few systems. It has also been observed in P3HS:PCBM, ${ }^{[26]}$ a selenophene analogue of P3HT, where thermal annealing causes the growth of a new transient feature at $820 \mathrm{~nm}$ in addition to the pre-existing $1150 \mathrm{~nm}$ localised polaron band. This new $820 \mathrm{~nm}$ feature was assigned to delocalised polarons that increase in population upon annealing, which is known to enhance the crystallinity of the blend film. This bimodal (delocalised and localised) polaron behaviour therefore may be more likely to occur in blends that have a mixture of both amorphous and crystalline domains. KP115 is known to have a moderate crystallinity, with a substantial in-plane long-axis orientation in neat films (which persists to a lesser extent in blend films) with disordered side chains. ${ }^{[10][35]}$ It is therefore reasonable to assume that, like P3HT:PCBM and P3HS:PCBM, both crystalline and amorphous phases are present in the KP115:PCBM blend film. This point, in addition to the similar bimodal band behaviour of the KP115:PCBM TAS spectrum, leads to the assignment of the feature around $750 \mathrm{~nm}$ to delocalised KP115 polarons while the broad band at approximately $1080 \mathrm{~nm}$ can be 
assigned to localised KP115 polarons. Note that photoinduced electroabsorption is also possible below at wavelengths below $700 \mathrm{~nm},{ }^{[36]}$ however this effect is unlikely to be large at these relatively long timescales. ${ }^{[37]}$

The charge carrier decay dynamics were measured prior to light soaking at probe wavelengths of $\lambda_{p r b}=720$ and $1000 \mathrm{~nm}$ (Figure 5), in order to differentiate between the delocalised and localised polarons respectively. These probe wavelengths were chosen in order to use the same photodetector (to avoid realignments) and, for the $720 \mathrm{~nm}$ probe, to be as close as possible to the delocalised polaron peak whilst avoiding ground state bleaching effects. Both probe wavelengths show an excitation-density dependent fast phase, prior to $\sim 3 \mu$ s for $\lambda_{\text {prb }}=$ $720 \mathrm{~nm}$ and $\sim 10 \mu \mathrm{s}$ for $\lambda_{p r b}=1000 \mathrm{~nm}$, while at longer times a power-law function can be fitted to the decay curves. The dependence on excitation density is shown in Figures S2-S6, Supporting Information. The fast phase shows an increasing rate of polaron decay with increasing excitation density, followed by a saturation regime: this is clear evidence of bimolecular recombination (Figures S2-S6). Indeed, no evidence of monomolecular behaviour was observed, thus geminate (monomolecular) recombination and triplet decay on these timescales can be discounted.

The decay kinetics during the power law phase of KP115:PCBM prior to light soaking showed different behaviour for the two probe wavelengths. At a probe wavelength of 1000 nm (Figure 5b) the power law $\alpha$ value of 0.43 is similar to what has been observed previously for P3HT:PCBM at probe wavelengths of $980-1000 \mathrm{~nm} \cdot{ }^{[20,26]}$ The kinetics at $1000 \mathrm{~nm}$ thus have a significant contribution from localised, trapped polarons. Thermal activation of these polarons out of the trap states is necessary before recombination can occur, thus causing a relatively slow recombination rate. At $720 \mathrm{~nm}$, where the freely mobile, delocalised polaron is expected to be the primary absorbing species, the power law phase exhibits fast decay kinetics, 
with an $\alpha$ of 0.68 for each excitation density (Figure 5a). Although not quite trap-free pure bimolecular recombination, consistent with what has previously been observed for P3HT:PCBM, ${ }^{[20]}$ these kinetics are significantly faster than those observed at longer probe wavelengths. This suggests that it is partially delocalised polarons that are undergoing bimolecular recombination at this probe wavelength or, alternatively, both delocalised and localised polarons are present, thus the kinetics are a combination of both trap-free and traplimited recombination respectively.

After light soaking (Figure 4b), the transient absorption spectrum shows no significant changes in overall shape on the microsecond timescale, with the features at 1080 and $<750$ $\mathrm{nm}$ still present and unshifted. A small drop in $\triangle O D$ at $1 \mu$ s is observed. A distinct alteration in the decay dynamics at $1000 \mathrm{~nm}$ is observed upon photodegradation: the kinetics of the trapped polarons are appreciably slower, with $\alpha$ decreasing from 0.43 to 0.13 (Figure $5 b$ ). This implies that the light degradation process has resulted in an increased trap depth, slowing down the polaron recombination at long times. The power law decay dynamics of the delocalised KP115 polaron at $720 \mathrm{~nm}$ do not show such a large change (Figure 5a), with the $\alpha$ value decreasing from 0.68 to 0.55 . This suggests that the bimolecular recombination of the delocalised free polymer polarons are much less affected by the light soaking process compared to the localised polarons at $1000 \mathrm{~nm}$, but the increased trap depth is still apparent.

The $\triangle O D$ (which is directly proportional to the charge carrier density) of the delocalised polaron band shows a small decrease with photodegradation prior to $2 \mu \mathrm{s}$ (from $1 \times 10^{-4}$ to $8 \mathrm{x}$ $10^{-5}$ at $\left.1 \mu \mathrm{s}\right)$. It has been previously established that the $\triangle O D$ at $1 \mu \mathrm{s}$ of a polymer:PCBM blend film is directly correlated with the $J_{S C}$ of the resultant photovoltaic device. ${ }^{[38]}$ According to this correlation, the small drop in $\triangle O D$ upon photodegradation would cause a reduction in 
the $J_{S C}$ of approximately $0.5 \mathrm{~mA} \mathrm{~cm} \mathrm{~cm}^{-2}$. The decrease in $J_{S C}$ observed in KP115:PCBM upon photodegradation is almost four times more than this. It is possible that a reduced charge carrier photogeneration yield is a partial contributor to the decreased $J_{S C}$. However, it appears that the loss in $J_{S C}$ can primarily be associated with the charge collection process, with an increase in trapping effects adversely influencing the efficiency of charge extraction. This is consistent with the $J V$ curves, which show that at high negative driving voltages, the same current density under illumination is present before and after photodegradation (Figure 2). This idea will be examined further by applying a variety of electrical charge transport techniques to the KP115:PCBM device.

\subsection{Time-resolved charge extraction using a nanosecond switch}

The charge extraction techniques complement TAS by providing information on charge carrier density, mobility and lifetime. In order to examine more closely the effects of photodegradation on the charge carrier density and decay dynamics, a time-resolved charge extraction (TRCE) technique was utilised. ${ }^{[9]}$ This involves a nanosecond time-resolved switch, where TRCE involves photogenerating charges with a nanosecond laser pulse in a photovoltaic device held at a very high impedance of $10 \mathrm{M} \Omega$ (open circuit), then switching to short circuit (low impedance) after an adjustable delay time, allowing charge extraction to occur under the influence of the built-in field.

Prior to light degradation, the charge carrier dynamics of KP115:PCBM show a clear biphasic behaviour (Figure 6a), as previously reported. ${ }^{[9]}$ The $\alpha$ values are similar to that observed in TAS, with an $\alpha$ of 0.35 prior to light soaking and an $\alpha$ of 0.2 afterwards. After the light soaking process, a small drop in charge carrier density after photodegradation is evident at early times (before $2 \mu$ s). After $\sim 2 \mu$ s the photodegraded sample charge carrier density 
decays more slowly, giving a greater charge carrier density at long times. This is also consistent with the TAS results.

The bimolecular recombination coefficient, $\beta$, as a function of $n$ (Figure $6 \mathbf{b}$ ) has been calculated from the gradient of the charge carrier decay in Figure 6a; this methodology also provides information on $\beta$ at long times:

$\beta(t)=-\frac{d n}{d t} \frac{1}{n^{2}}$

Prior to light degradation, a region where $\beta$ is strongly dependent on $n$ is observed (as reported previously ${ }^{[9]}$ ), followed by a saturation $n$-independent regime at a $\beta$ of $8 \times 10^{-12} \mathrm{~cm}^{3} \mathrm{~s}^{-}$

${ }^{1}$. After light degradation, $\beta$ is virtually unchanged at high charge carrier densities, implying the photodegradation has not affected the recombination of the free charge carriers. At low charge densities, however, $\beta$ is considerably lower after light soaking (by over an order of magnitude), indicating a greater proportion of deeply trapped charge carriers unable to participate in bimolecular recombination.

\subsection{Photovoltage decay}

The decay of the photovoltage $\left(V_{O C}\right)$ over time can be examined (Figure 7a) and the voltages at each time correlated with the measured charge carrier densities from the TRCE measurements (Figure 7b). The voltage decay shows that at early times, the control sample has a slightly higher $V_{O C}$ (by $\sim 10 \mathrm{mV}$ ). However, a crossover occurs at $\sim 10 \mu$ s such that at longer times the photodegraded sample has a higher photovoltage. The measurement of photovoltage and charge carrier density at each time delay allows the relationship between 
these two parameters to be examined (Figure 7b). The device shows very different behaviour before and after light soaking, where photodegradation induces a weaker dependence of charge density on $V_{O C}$. Doubling the charge density from $2 \times 10^{16} \mathrm{~cm}^{-3}$ to $4 \times 10^{16} \mathrm{~cm}^{-3}$ produces a larger change in voltage in the photodegraded sample. This is directly correlated with the density of states distribution, ${ }^{[39]}$ with the data following the approximate exponential relationship of $n=n_{0} e^{\gamma V o c}$, where $n_{0}$ is the average charge density in the dark and $\gamma$ is the slope. Bimolecular recombination in a trap-free medium is expected to have $\gamma=e / 2 k_{B} T \sim 19$ at room temperature. The slope $\gamma$ for $\mathrm{KP} 115: \mathrm{PCBM}$ is 13 prior to light soaking (similar to P3HT:PCBM ${ }^{[39]}$ ) but drops to only 7 afterwards. This suggests that after photodegradation the DOS distribution widens, in turn implying a more prominent exponential tail of trap states.

\subsection{Photo-CELIV}

Photo-induced charge extraction by linearly increasing voltage (photo-CELIV) is used to estimate charge carrier mobility. ${ }^{[40-42]}$ Photogenerated charges are extracted after an adjustable delay time by a linearly increasing voltage pulse, $A=\Delta U / \Delta t$. The mobility is obtained from the time at which the maximum photocurrent response, $t_{\max }$, occurs:

$$
\mu=\frac{2 d^{2}}{3 A t_{\max }^{2}\left[1+0.36 \frac{\Delta j}{j(0)}\right]}
$$

Where $d$ is the active layer thickness, $A$ characterises the voltage pulse as $\Delta U / \Delta t, \Delta j$ is the photogenerated current response and $j(0)=A C$ (the capacitive current response), where $\Delta j<<j(0)$ is necessary. However, the $R C$ constant of the system can limit the accuracy of this 
technique, particularly if thin film photovoltaic devices with high charge carrier mobility are used.

In order to circumvent $R C$ limitations, the photo-CELIV curves were measured using devices with a small area $\left(5 \mathrm{~mm}^{2}\right.$, giving a capacitance of $\left.\sim 7 \times 10^{-10} \mathrm{~F}\right)$. Furthermore, the nanosecond time-resolved switch was employed. The operation of this switch in photo-CELIV involves photogenerating charges in a photovoltaic device held at high impedance (open circuit), then switching to short circuit (low impedance) after an adjustable delay time, simultaneously applying the triangular voltage pulse to extract the charges. The advantage of using such a method for photo-CELIV is that the high impedance of the circuit prior to application of the voltage pulse (and closing of the switch) causes the normal photocurrent response prior to $t=0$ to be reduced to almost zero ( $\sim \mathrm{nA}$ range). The charge leakage is therefore negligible and $V_{O C}$ conditions are present. This is of particular value if the built-in potential cannot be completely compensated for by applying an offset voltage, ${ }^{[43]}$ as in the case of KP115:PCBM. It therefore also allows a more accurate determination of extracted charge carrier density since the charge loss during the delay time between the laser pulse and closing the switch is solely due to recombination, rather than possessing an additional contribution from charge leakage.

The photo-CELIV results are shown in Figure 8. The mobilities calculated from equation 2 are shown in the inset of Figure 8 . Prior to light degradation, the calculated mobility is $\sim 8 \mathrm{x}$ $10^{-4} \mathrm{~cm}^{2} \mathrm{~V}^{-1} \mathrm{~s}^{-1}$; this value is higher than that previously published. ${ }^{[10]}$ This could be because in this study the device area was reduced by a factor of three (to $\sim 5 \mathrm{~mm}^{2}$ ) in order to minimise $R C$ limitations and better resolve $t_{\max }$. Furthermore, the devices measured here have much higher efficiencies $\left(4.4 \%\right.$ rather than the previous $\left.3.0 \%{ }^{[10]}\right)$. The nanosecond switch does introduce a small artifact, however: a small capacitive discharge prior to $t=0$. The photoCELIV curves were therefore also measured without the switch to check for reproducibility 
between techniques. Similar mobility values were determined with and without the nanosecond switch, as shown in the Figure 8 inset.

After light degradation, the photo-CELIV curves change: most notably, the quantity of charge extracted has increased by a factor of 2.3 at this delay time of $100 \mu \mathrm{s}$. The increase in charge at these long times is consistent with the TAS and TRCE results, which show greater $\triangle O D$ and $n$ values respectively at long times after light soaking. Given the slower trap-limited bimolecular recombination after photodegradation, more charge carriers survive to long times.

Furthermore, the mobility decreases very slightly to $\sim 6 \mathrm{x} 10^{-4} \mathrm{~cm}^{2} \mathrm{~V}^{-1} \mathrm{~s}^{-1}$ after photodegradation; this decrease is largely due to the increase in $\Delta j$ (from 15.2 to $17.9 \mathrm{~mA} . \mathrm{cm}^{-}$ ${ }^{2}$ ), while the $t_{\max }$ only increases from 605 to $670 \mathrm{~ns}$. Lowering $A$ did not substantially increase this difference. The two mobilities are very similar within experimental error. Indeed, the mobility over time (as shown in the inset of Figure 8) also shows no evidence of a decrease, either before or after light soaking. These are surprising results, as it would be expected that if the light-soaked device were subject to an increase in trapping effects - as suggested by the higher charge carrier densities at long times shown by photo-CELIV, TRCE and TAS - this would manifest in a substantially lower charge carrier mobility. Furthermore, only deeply trapped charges usually remain at long delay times and this would also be expected to decrease the mobility. Indeed, Eng et al. ${ }^{[18]}$ predicted a decrease in charge carrier mobility of over two orders of magnitude from the nanosecond to millisecond timescales for P3HT:PCBM. This is clearly not observed here. However, it is important to note that given the photon density incident on the active device area (and assuming a photon to charge conversion of unity), the photo-CELIV technique is extracting less than $20 \%$ of the total photogenerated charge carriers. This is not solely due to truncation of the voltage pulse at 5 $\mu$ s: the TRCE technique extracts over $60 \%$ of the total photogenerated charges under the 
same integration conditions ( $5 \mu$ s after opening of the switch). This implies that in the photoCELIV measurement some charges are also recombining within the duration of the laser pulse or during the actual extraction process. Another possible explanation is that a large proportion of photogenerated charge carriers remain trapped and are not extracted, as seen by the small asymptotic tail of charge that does not reach the capacitive response. As such, the lower mobility of deeply trapped charges may not be observable using photo-CELIV. It is likely that it is primarily the free, delocalised charges that are being extracted here, hence the relatively high mobility.

\subsection{Photocurrent decay}

The photocurrent decay was measured by applying a $0.4 \mathrm{~V}$ constant reverse bias to the device under low-impedance conditions and low laser excitation density. The results are shown in Figure 9. The photocurrent decay is $R C$ limited prior to $\sim 350 \mathrm{~ns}$, after which time a reservoir of charge carriers is present, as expected for a non-Langevin system. ${ }^{[10]}$ Since only charge equal to $C U_{0}$ can be extracted per transit time and bimolecular recombination is relatively slow in KP115:PCBM, accumulation of charge carriers in the active layer occurs. After $\sim 1 \mu \mathrm{s}$ the reservoir is progressively depleted by extraction, and very little charge is left after $10 \mu \mathrm{s}$. The extraction time is limited by trapping, inducing a tail of charge after $\sim 2 \mu \mathrm{s}$. The extraction that is occurring on the sub-microsecond and early microsecond timescale is that of the free delocalised charges prior to any thermalisation into the sub-bandgap (trapped) states, whereas extraction on the longer timescales - in the tail of the current transient - is of charge carriers de-trapped from these localised states. After light soaking, however, a larger proportion of charges are localised into the sub bandgap states. As such, the tail of charge in Figure 9 is significantly more prominent, showing that the charge extraction process occurs on much longer timescales after light soaking. At the low charge densities present after $2 \mu \mathrm{s}$, 
the low internal resistance consequently present in the cell implies that conditions closer to $J_{S C}$ (rather than $V_{O C}$ ) are present. This implies that the more significant trapping after light soaking also would have an effect on $J_{S C}$.

\section{Discussion}

The transient absorption measurements revealed a bimodal KP115 polaron population, comprised of both delocalised charge carriers with relatively fast bimolecular recombination kinetics and localised (trapped) charge carriers with considerably slower - trap-limited decay. After light soaking, the localised polaron decay dynamics slow down, suggesting the presence of energetically deeper trap states, while the delocalised polaron kinetics are less affected. This results in a higher concentration of charges at long times, a result also observed using photo-CELIV, photocurrent decay and time-resolved charge extraction. This TRCE technique also allows the photovoltage decay over time to be monitored, allowing the charge density as a function of photovoltage to be examined. The results of this analysis suggest that the density of states broadens after photodegradation. Photodegradation therefore appears to result in an alteration in the bimodal KP115 polaron population, with the polarons becoming trapped in a broader, deeper density of localised states (as illustrated in Figure 10). As such, after photo-degradation the proportion of charge carriers influenced by thermalisation into these sub-bandgap states increases. This consequently slows down recombination - leading to the higher charge densities observed at long times using each technique - but also inhibits charge extraction. It is likely that this loss in charge extraction efficiency is the main contributor to the decrease in $J_{S C}$ observed after light soaking.

Another piece of evidence for the significant role that enhanced charge carrier trapping plays in the decrease in $J_{S C}$ after photodegradation is a consideration of the effect of active layer 
thickness. In a thin device, charge carriers are more likely to be extracted prior to trapping. ${ }^{[44]}$ In a thicker device, however, the average distance charges must travel before reaching the contacts increases, thus more charge carrier localisation would occur prior to extraction. After light-soaking such a thick device, the enhancement in charge carrier trapping that occurs suggests that charge extraction would be even more strongly inhibited. In this thicker device case, the $J_{S C}$ - and efficiency - would be expected to decrease more strongly and indeed this is what has been observed (Figure 11). This observation is also a clear indication that the photodegradation impacts primarily on the active layer rather than the contacts. Furthermore, P3HT:PCBM reference devices constructed with the same contacts, HIL, and EIL show quite different (slower) photodegradation behaviour. Thus it can be presumed that light soaking has a minimal effect on the contacts under these conditions, and that it is predominantly the active layer that undergoes the changes in trapping induced by light soaking. This conclusion is supported by the TAS results, where identical results were observed upon light soaking with both devices and simple films coated onto glass (encapsulated, but with no contacts or interlayers).

Charge carrier trapping is typically associated with a concomitant decrease in charge carrier mobility, which is not observed here. ${ }^{[45-47]}$ This is possibly due to the inability of the photoCELIV technique to monitor all trapped charges present in this device under these experimental conditions. Extraction of deeply trapped charges is not observed as a peak with a clearly defined $t_{\max }$ : a long tail of extracted charge carriers is present instead, which is truncated by the end of the applied voltage pulse. Thus the low mobilities of deeply trapped charges are not able to be measured using this technique. Any differences in charge carrier mobility after photodegradation would be expected to arise from those carriers trapped in the deepest states. 
The higher extracted charge density and longer charge carrier lifetime present at long times after photodegradation appears to be inconsistent with the reduction in $J_{S C}$. There are two possible reasons why this is not the case. Firstly, the crossover in charge density at $2 \mu \mathrm{s}$ consistently observed in the TAS, TRCE and photocurrent decay measurements may imply that the charge carrier density is lower after photodegradation at much earlier times (prior to the resolution of the techniques employed here). In this scenario, fast charge carrier extraction on the sub-microsecond timescale would be an important contributor to the $J_{S C}$. Secondly, it must be recalled that these transient techniques are usually more reminiscent of $V_{O C}$ conditions (where bimolecular recombination dominates ${ }^{[48]}$ ) rather than $J_{S C}$. Since the deeper, broader trap states present after photodegradation enhance charge carrier trapping, bimolecular recombination of these trapped charges at $V_{O C}$ is inhibited. However, the consequently higher charge carrier density at long times after photodegradation will not necessarily be present under $J_{S C}$ conditions, where bimolecular recombination is less prevalent. Instead, it has been suggested that close to $J_{S C}$, the low charge densities lead to monomolecular trap-induced recombination, which could be the primary loss mechanism. ${ }^{[49]}$ It is probable, therefore, that the deeper, broader trap states present after photodegradation slow down bimolecular recombination under open circuit conditions (boosting charge density at long times), but under the low charge density conditions of $J_{S C}$ actually promote recombination. A higher level of trap-induced recombination at short circuit, thereby reducing the efficiency of charge extraction, would account for the loss of $J_{S C}$ after photodegradation.

Short circuit conditions are difficult to probe experimentally using transient techniques, particularly in these types of solar cells which have relatively high external quantum efficiencies $(>70 \%)$. The efficient charge extraction that occurs under the applied electric field at $J_{S C}$ allows only low charge densities to remain, which can be difficult to measure with any level of sensitivity. Another issue is the difference between transient and steady-state 
techniques. In a transient measurement, a high number of photons are incident on the solar cell for a very short period of time ( $8 \mathrm{~ns}$ in the above results), and all charge carriers recombine or are extracted before the second pulse occurs; the system is allowed to return to equilibrium (its ground state). Steady state measurements, on the other hand, rely on a constant light source that induces a steady state population of charge. Results can vary because of this: the charge carrier lifetime measured by transient photovoltage (which, as a small perturbation technique, approximates steady-state) may differ substantially from that measured by photo-CELIV, for instance.

However, despite these issues of $J_{S C}$ versus $V_{O C}$ and transient versus steady state conditions, the deeper, broader density of trap states that lead to enhanced charge carrier trapping after light soaking have been observed here using several different experimental techniques and conditions. The detrimental effect this enhanced charge carrier trapping has on $J_{S C}$ is also supported by the thickness dependent light soaking results, where charge carrier trapping is expected to have a greater effect in thicker devices, as confirmed by the larger photodegradation effect. Furthermore, the observation in the $J V$ curves that the photocurrent of the light soaked cell approaches that of the control device at high negative bias is also indicative of a trapping effect. At such high driving forces, the extraction time is very fast and thus extraction occurs before any trap-assisted recombination takes place: the control and light soaked solar cells therefore produce the same photocurrent. At $J_{S C}$ a smaller driving force is present, therefore the trap-assisted recombination mechanism competes more effectively with charge extraction after light soaking.

Relatively little is known about the nature of these localised trap states and the exact chemical reaction pathways and structural or conformational changes that are required to induce charge carrier trapping. The fullerene or impurities may also play an important role in trap formation. 
Furthermore, it is likely that the mechanism of trap formation, resultant nature of the localised states, and consequent effect on device performance is highly dependent on chemical structure. For example, the PCDTBT photodegradation mechanism (in air) has been reported to involve photo-oxidative scission of a C-N bond in the carbazole unit. ${ }^{[7]} \mathrm{KP} 115$ has a very different chemical structure and its specific photodegradation mechanism needs to be examined in detail in order to ascertain the best methodology to inhibit these detrimental photodegradation effects. Photodegradation, even in encapsulated devices, and charge carrier trapping are clearly significant issues that need further research and must be addressed for successful commercialisation.

\section{Conclusions}

A photodegradation study has been performed on KP115:PCBM photovoltaic devices. This process induces a significant decrease in power conversion efficiency, primarily as a result of a loss in $J_{S C}$. The initial stage of this photodegradation process, the burn-in phase, has been examined using a combination of transient absorption spectroscopy and charge extraction measurements. It appears that the main reason for the loss in $J_{S C}$ upon photodegradation is related to the efficiency of charge transport and extraction, although a decrease in charge carrier density at early times may also contribute. The bimodal polymer polaron population evident from TAS has both delocalised and localised, trapped polarons. After light soaking, the distribution of charge carrier trap states becomes broader with consequently energetically deeper traps. As such, after photodegradation the proportion of charge carriers influenced by thermalisation into these sub-bandgap states increases.

Under short laser pulse and $V_{O C}$ conditions this enhanced trapping means that charge carriers are able to postpone bimolecular recombination for longer, leading to higher charge densities 
at long times. Under the low charge density and constant illumination conditions of $J_{S C}$, however, bimolecular recombination is less dominant and the primary recombination mechanism appears to be trap-induced recombination. After light soaking this loss mechanism competes more effectively with charge extraction due to the deeper, broader trap states present. The $J_{S C}$ decreases as a result of this reduction in charge extraction efficiency. This conclusion is supported by the observation that photodegradation effects are greater for thicker active layers. In thicker devices more charge carrier localisation would occur prior to extraction. After light soaking, therefore, the enhanced charge carrier trapping causes charge carrier extraction to be impeded more strongly and thus $J_{S C}$ decreases by a larger fraction.

In conclusion, therefore, an in-depth photodegradation study of KP115:PCBM has shown that light soaking induces a deeper, broader density of localised (trapped) states. The enhanced charge carrier trapping influences the recombination characteristics of the active layer, reducing the efficiency of charge extraction under short circuit conditions.

\section{Experimental section}

Devices: Devices of KP115:PCBM (1:2 by weight) were fabricated using the same method as Peet et $a l .{ }^{[7]}$ with an inverted Ag/hole-injecting layer (HIL)/active layer/ electron-injecting layer (EIL)/ITO structure, where the HIL and EIL are Konarka proprietary materials. The active layer thickness was $140 \mathrm{~nm}$ with an active area of $\sim 5 \mathrm{~mm}^{2}$. Devices were fabricated in air, then transferred to a glovebox for glass encapsulation.

Photo-degradation: The long-term photodegradation was accomplished on non-encapsulated devices inside a glove box using a cold LED array set to 1 sun. The short-term burn-in photodegradation for the TAS and charge extraction measurements was done on encapsulated 
devices in ambient air using a solar simulator set to 1 sun and fitted with a water filter to absorb heat.

Nanosecond transient absorption spectroscopy: The third harmonic (355 nm) of a Nd:YAG laser after pulse compression (Ekspla, SL311) was employed for excitation. The repetition rate of the laser was $10 \mathrm{~Hz}$ and the pulse duration was about $150 \mathrm{ps.} \mathrm{A} \mathrm{Xe} \mathrm{flash} \mathrm{lamp}$ (Hamamatsu, L4642, 2-1s pulse duration) was used as a probe light source. The probe light was directed into a Si photodiode (New Focus, 1601). For near-infrared wavelength measurements, an InGaAs photodetector (New Focus, 1611) was used. The signal from the detector was introduced into a digital oscilloscope (LeCroy, 6200 A). With this system, we could measure a small absorbance change $\left(<10^{-3}\right)$ after 100 pulses, on average. The rise time of the overall system was about 400 ps. The intensity of the laser pulse was measured with a pyroelectric energy meter (OPHIR, PE25-SH-V2). All measurements were carried out at room temperature.

Microsecond-millisecond transient absorption spectroscopy: The encapsulated devices were excited in transmission mode by a laser pulse $(6 \mathrm{~ns}, 532 \mathrm{~nm}$, repetition rate $10 \mathrm{~Hz})$ from a Nd:YAG laser (Spectra-Physics, INDI-40-10) with a pump wavelength of $532 \mathrm{~nm}$, using pump intensities from 0.03 to $10 \mu \mathrm{J} . \mathrm{cm}^{-2}$ and a repetition frequency of $1 \mathrm{~Hz}$. The Xe probe lamp (Edinburgh Instruments, Xe900) with a stabilised power supply, with a typical probe wavelengths of 720 and $1000 \mathrm{~nm}$, adjustable using a monochromator. The probe light passing through the device was detected with a silicon (Femto, HCA-S-200M-SI) or an InGaAs photodiode (Femto, HCA-S-200M-IN). The signal from the photodiode was amplified (Femto, DHPVA-200) and collected with a digital oscilloscope (Tektronics, DPO4054), which was synchronised with a trigger signal of the pump laser pulse from a photodiode (Newport, 818- 
BB-40). To reduce stray light, scattered light, and sample emission, appropriate optical cut-off and bandpass filters were placed before and after the sample.

Photo-CELIV measurements: The device was illuminated by the laser pulse as above, but in reflection mode. After an adjustable delay time controlled by a digital delay generator (Stanford Research DG535), photo-generated charges were extracted using a linearly increasing voltage pulse applied by a function generator. The current transients were recorded using an oscilloscope (50 Ohm input impedance). The time resolution of the setup is around 5 ns, and the calculated $R C$ constant was $\sim 250 \mathrm{~ns}$.

Time-resolved charge extraction: The device, held at open circuit, was illuminated by the laser pulse as above. After an adjustable delay time, extraction of the photogenerated charges under the built-in field was accomplished by switching to short circuit using a nanosecond time-resolved switch. The photocurrent was then integrated to ascertain the charge density at each delay time.

Photovoltage decays: The device, held at open circuit, was illuminated by the laser pulse and then the decay of photogenerated voltage was monitored over time.

\section{Supporting Information}

Supporting Information is available online from the Wiley Online Library or from the author.

\section{Acknowledgements}

This research was supported under the Australian Research Council's Linkage Projects funding scheme and by Konarka Technologies. AJM acknowledges the ARC for providing equipment support through LIEF, as well as supporting AJM and TMC with an Australian Research Fellowship and Australian Postdoctoral Fellowship Industry, respectively.

Received: ((will be filled in by the editorial staff))

Revised: ((will be filled in by the editorial staff))

Published online: ((will be filled in by the editorial staff)) 


\section{References}

[1] Z. He, C. Zhong, S. Su, M. Xu, H. Wu, Y. Cao, Nat Photon 2012, 6, 591.

[2] M. Jørgensen, K. Norrman, S. A. Gevorgyan, T. Tromholt, B. Andreasen, F. C. Krebs, Adv. Mater. 2012, 24, 580 .

[3] M. Jørgensen, K. Norrman, F. C. Krebs, Sol. Energy Mater. Sol. Cells 2008, 92, 686.

[4] C. H. Peters, I. T. Sachs-Quintana, W. R. Mateker, T. Heumueller, J. Rivnay, R. Noriega, Z. M. Beiley, E. T. Hoke, A. Salleo, M. D. McGehee, Adv. Mater. 2012, 24, 663.

[5] T.-Y. Chu, S. Alem, S.-W. Tsang, S.-C. Tse, S. Wakim, J. Lu, G. Dennler, D. Waller, R. Gaudiana, Y. Tao, Appl. Phys. Lett. 2011, 98, 253301.

[6] S. H. Park, A. Roy, S. Beaupre, S. Cho, N. Coates, J. S. Moon, D. Moses, M. Leclerc, K. Lee, A. J. Heeger, Nat. Photonics 2009, 3, 297.

[7] A. Tournebize, P.-O. Bussière, P. Wong-Wah-Chung, S. Thérias, A. Rivaton, J.-L. Gardette, S. Beaupré, M. Leclerc, Adv. Energy Mater. 2012, n/a.

[8] M. O. Reese, A. J. Morfa, M. S. White, N. Kopidakis, S. E. Shaheen, G. Rumbles, D. S. Ginley, Sol. Energy Mater. Sol. Cells 2008, 92, 746.

[9] T. M. Clarke, J. Peet, P. Denk, G. Dennler, C. Lungenschmied, A. J. Mozer, Energ. Environ. Sci. 2012, 5, 5241.

[10] T. M. Clarke, D. B. Rodovsky, A. A. Herzing, J. Peet, G. Dennler, D. DeLongchamp, C. Lungenschmied, A. J. Mozer, Adv. Energy Mater. 2011, 1, 1062.

[11] J. Peet, L. Wen, P. Byrne, S. Rodman, K. Forberich, Y. Shao, N. Drolet, R. Gaudiana, G. Dennler, D. Waller, Appl. Phys. Lett. 2011, 98, 1.

[12] S. Subramaniyan, H. Xin, F. S. Kim, S. Shoaee, J. R. Durrant, S. A. Jenekhe, Adv. Energy Mater. 2011, 1, 854.

[13] M. Zhang, X. Guo, Y. Li, Adv. Energy Mater. 2011, 1, 557. 
[14] E. T. Hoke, I. T. Sachs-Quintana, M. T. Lloyd, I. Kauvar, W. R. Mateker, A. M. Nardes, C. H. Peters, N. Kopidakis, M. D. McGehee, Adv. Energy Mater. 2012, 2, 1351.

[15] T. Erb, U. Zhokhavets, G. Gobsch, S. Raleva, B. Stühn, P. Schilinsky, C. Waldauf, C. J. Brabec, Adv. Funct. Mater. 2005, 15, 1193.

[16] Y. Kim, S. Cook, S. M. Tuladhar, S. A. Choulis, J. Nelson, J. R. Durrant, D. D. C. Bradley, M. Giles, I. Mcculloch, C.-S. Ha, M. Ree, Nat. Mater. 2006, 5, 197.

[17] T. Clarke, A. Ballantyne, F. Jamieson, C. Brabec, J. Nelson, J. Durrant, Chem. Commun. 2009, 89.

[18] M. P. Eng, P. R. F. Barnes, J. R. Durrant, J. Phys. Chem. Lett. 2010, 1, 3096.

[19] J. Guo, H. Ohkita, H. Benten, S. Ito, J. Am. Chem. Soc. 2009, 131, 16869.

[20] J. Guo, H. Ohkita, S. Yokoya, H. Benten, S. Ito, J. Am. Chem. Soc. 2010, 132, 9631.

[21] H. Ohkita, S. Cook, Y. Astuti, W. Duffy, S. Tierney, W. Zhang, M. Heeney, I. McCulloch, J. Nelson, D. D. C. Bradley, J. R. Durrant, J. Am. Chem. Soc. 2008, 130, 3030.

[22] A. Ruseckas, M. Theander, M. R. Andersson, M. Svensson, M. Prato, O. Inganas, V. Sundstrom, Chem. Phys. Lett. 2000, 322, 136.

[23] A. Saeki, S. Seki, Y. Koizumi, S. Tagawa, J. Photochem. Photobiol. A: Chem. 2007, $186,158$.

[24] S. Shoaee, M. P. Eng, E. Espildora, J. L. Delgado, B. Campo, N. Martin, D. Vanderzande, J. R. Durrant, Energ. Environ. Sci. 2010, 3, 971.

[25] S. Yamamoto, H. Ohkita, H. Benten, S. Ito, J. Phys. Chem. C 2012, 116, 14804.

[26] T. M. Clarke, F. C. Jamieson, J. R. Durrant, J. Phys. Chem. C 2009.

[27] T. M. Clarke, A. M. Ballantyne, S. Tierney, M. Heeney, W. Duffy, I. McCulloch, J. Nelson, J. R. Durrant, J. Phys. Chem. C 2010, 114, 8068.

[28] I. Montanari, A. F. Nogueira, J. Nelson, J. R. Durrant, C. Winder, M. A. Loi, N. S. Sariciftci, C. Brabec., Appl. Phys. Lett. 2002, 81, 3001. 
[29] A. F. Nogueira, I. Montanari, J. Nelson, J. R. Durrant, C. Winder, N. S. Sariciftci, C. Brabec, J. Phys. Chem. B 2003, 107, 1567.

[30] J. Nelson, Phys. Rev. B 2003, 67, 155209.

[31] O. J. Korovyanko, R. Österbacka, X. M. Jiang, Z. V. Vardeny, R. A. J. Janssen, Phys. Rev. B 2001, 64, 235122.

[32] R. Österbacka, C. P. An, X. M. Jiang, Z. V. Vardeny, Science 2000, 287, 839.

[33] M. Westerling, R. Österbacka, H. Stubb, Phys. Rev. B 2002, 66, 165220.

[34] D. R. Lawson, D. L. Feldhiem, C. A. Foss, P. K. Dorhout, C. M. Elliott, C. R. Martin, B. Parkinson, J. Phys. Chem. 1992, 96, 7175.

[35] Dean DeLongchamp and Deanna Rodovsky, "Structural Measurements of Blend Films for Organic Photovoltaics", Materials Research Society Fall Meeting, Boston, USA, November 2010.

[36] C. X. Sheng, M. Tong, S. Singh, Z. V. Vardeny, Phys. Rev. B 2007, 75, 085206.

[37] E. W. Snedden, A. P. Monkman, F. B. Dias, J. Phys. Chem. C 2012, 116, 86.

[38] T. M. Clarke, A. Ballantyne, S. Shoaee, Y. W. Soon, W. Duffy, M. Heeney, I. McCulloch, J. Nelson, J. R. Durrant, Adv. Mater. 2010, 22, 5287.

[39] A. Maurano, R. Hamilton, C. G. Shuttle, A. M. Ballantyne, J. Nelson, B. O'Regan, W. Zhang, I. McCulloch, H. Azimi, M. Morana, C. J. Brabec, J. R. Durrant, Adv. Mater. 2010, 22, 4987.

[40] A. J. Mozer, G. Dennler, N. S. Sariciftci, M. Westerling, A. Pivrikas, R. Österbacka, G. Juška, Phys. Rev. B 2005, 72, 035217.

[41] A. J. Mozer, N. S. Sariciftci, A. Pivrikas, R. Österbacka, G. Juška, L. Brassat, H. Bässler, Phys. Rev. B 2005, 71, 035214.

[42] A. Pivrikas, N. S. Sariciftci, G. Juška, R. Österbacka, Prog. Photovoltaics 2007, 15, 677. 
[43] A. Baumann, J. Lorrmann, D. Rauh, C. Deibel, V. Dyakonov, Adv. Mater. 2012, 24, 4381.

[44] D. Moses, J. Wang, G. Yu, A. J. Heeger, Phys. Rev. Lett. 1998, 80, 2685.

[45] J. Cabanillas-Gonzalez, T. Virgili, A. Gambetta, G. Lanzani, T. D. Anthopoulos, D. M. de Leeuw, Phys. Rev. Lett. 2006, 96, 106601.

[46] A. Devizis, K. Meerholz, D. Hertel, V. Gulbinas, Phys. Rev. B 2010, 82, 155204.

[47] A. Devižis, A. Serbenta, K. Meerholz, D. Hertel, V. Gulbinas, Phys. Rev. Lett. 2009, $103,027404$.

[48] S. R. Cowan, A. Roy, A. J. Heeger, Phys. Rev. B 2010, 82, 245207.

[49] R. A. Street, M. Schoendorf, A. Roy, J. H. Lee, Phys. Rev. B 2010, 81, 205307. 


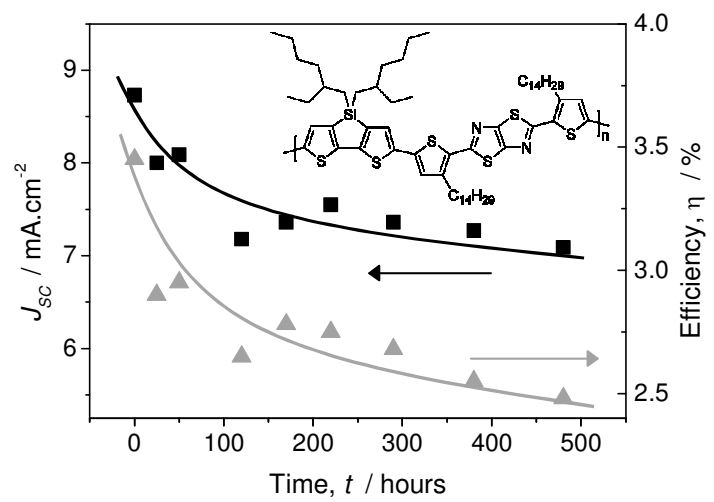

Figure 1. Long time degradation behaviour of KP115:PCBM photovoltaic devices under continuous white light (one sun equivalent) illumination in a glove-box, showing the initial burn-in phase prior to 100 hours and an approximately linear decay thereafter. The solid lines are guides for the eye. The inset shows the structure of KP115.

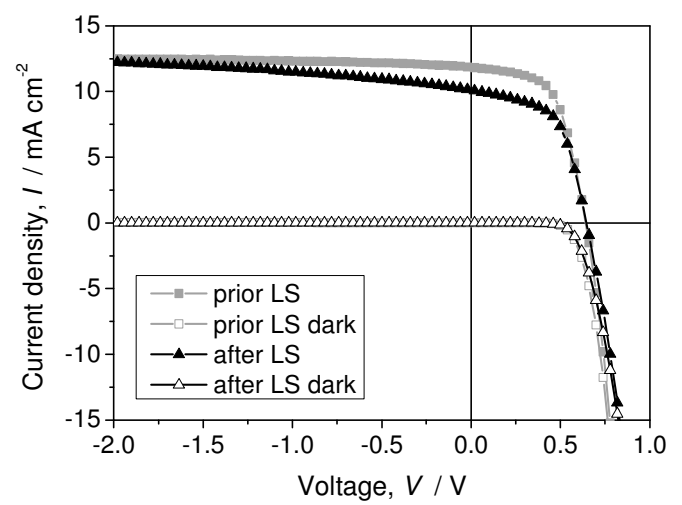

Figure 2. $J V$ curves of the encapsulated KP115:PCBM (1:2) photovoltaic device before and after 72 hours of light soaking (LS) at 1 sun. 


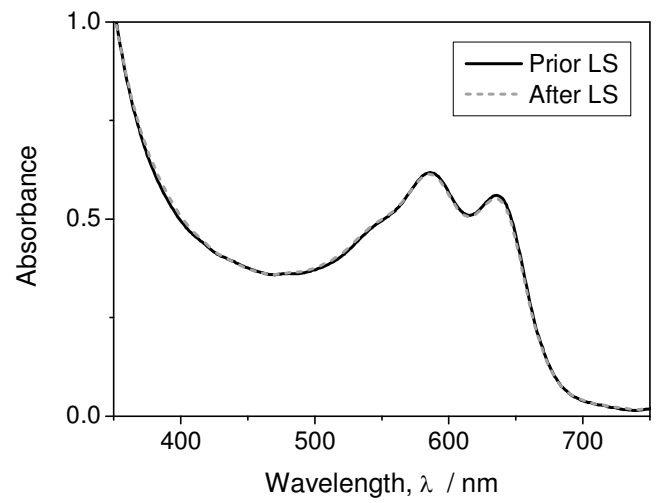

Figure 3. The ground state absorbance spectrum of the encapsulated KP115:PCBM (1:2) photovoltaic blend film before and after 72 hours of light soaking (LS). 

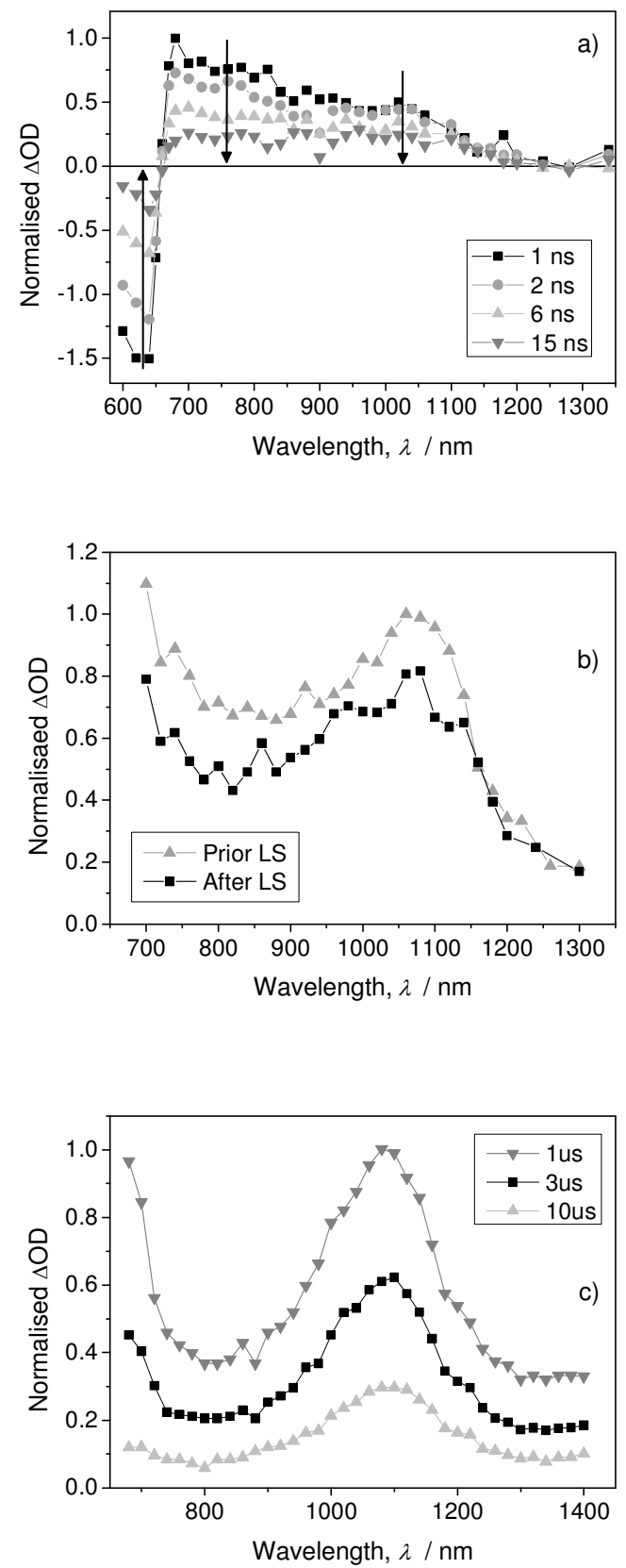

Figure 4. a) The transient absorption spectra of the non-degraded $\mathrm{KP} 115: \mathrm{PC}_{60} \mathrm{BM}$ device on the early nanosecond timescale. The microsecond-timescale transient absorption spectra of (b) $\mathrm{KP} 115: \mathrm{PC}_{60} \mathrm{BM}$ at $1 \mu \mathrm{s}$ before and after 72 hours of light soaking (LS) and (c) $\mathrm{KP} 115: \mathrm{PC}_{70} \mathrm{BM}$ encapsulated films in transmission, all using $10 \mu \mathrm{J} . \mathrm{cm}^{-2} 532 \mathrm{~nm}$ excitation. These results were reproducible over several samples and almost identical results were measured for devices. 

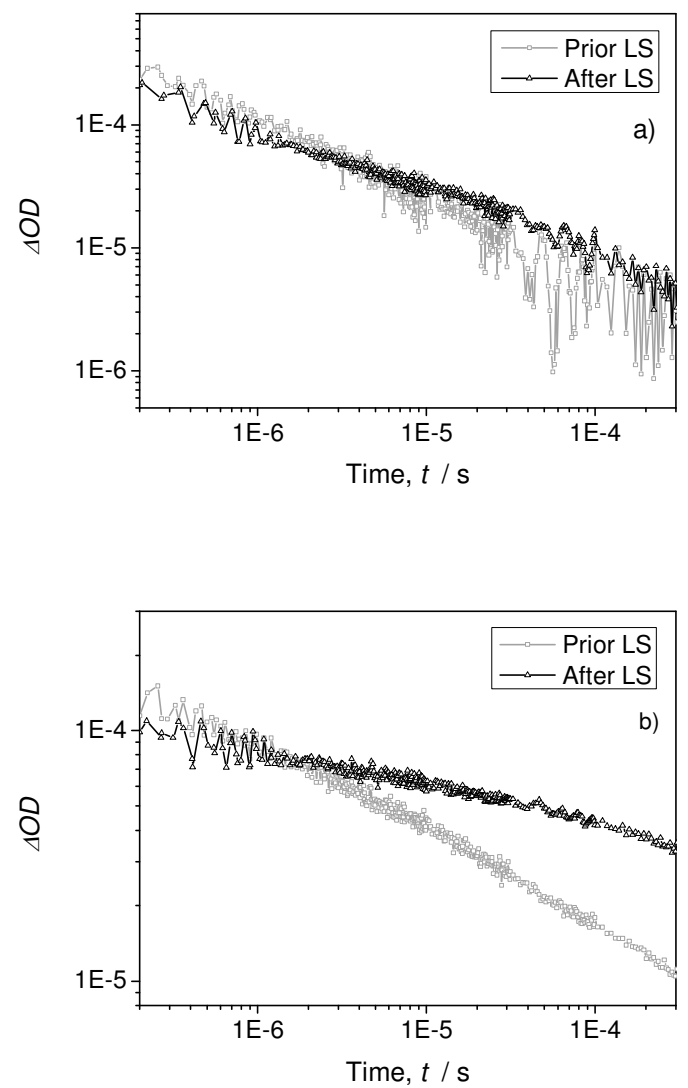

Figure 5. The transient absorption decays before and after 72 hours of light soaking (LS) of the encapsulated KP115:PCBM (1:2) photovoltaic device using $10 \mu \mathrm{J} . \mathrm{cm}^{-2} 532 \mathrm{~nm}$ excitation with probe wavelengths of a) $720 \mathrm{~nm}$ and b) $1000 \mathrm{~nm}$. 

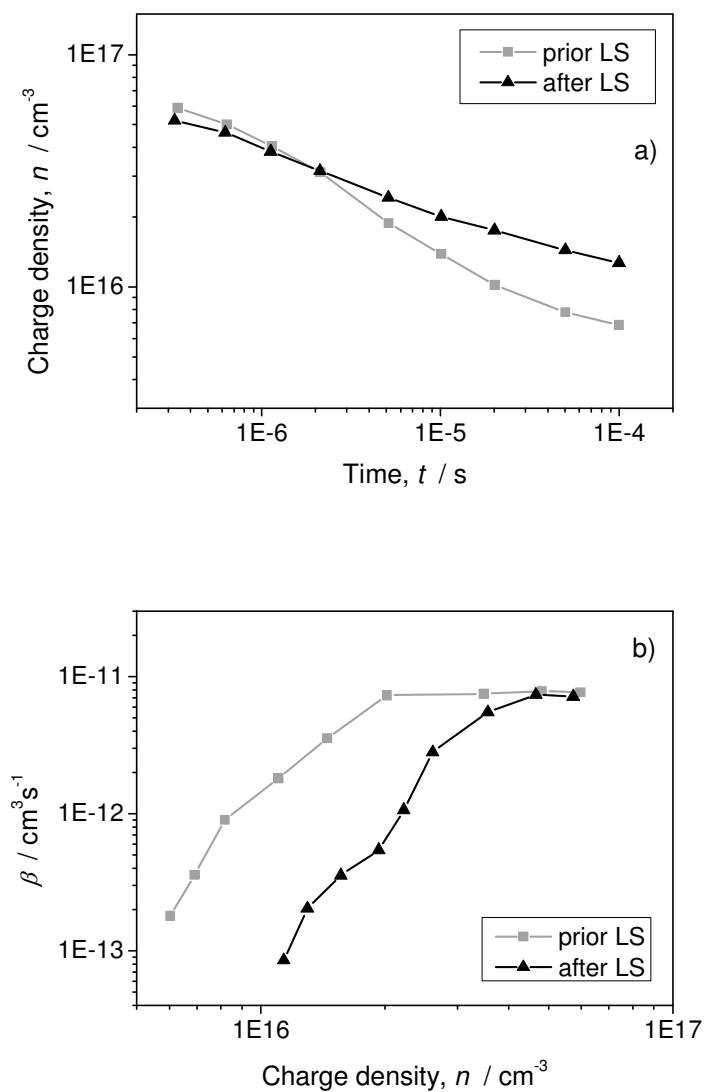

Figure 6. (a) Charge density decay with time of the encapsulated KP115:PCBM (1:2) photovoltaic device before and after light soaking (LS) $\left(\lambda_{\text {exc }}=532 \mathrm{~nm}, 0.3 \mu \mathrm{J} \mathrm{cm}^{-2}\right)$, measured using a nanosecond switch. (b) The bimolecular recombination coefficient $\beta$ as a function of charge carrier density before and after light-soaking. 

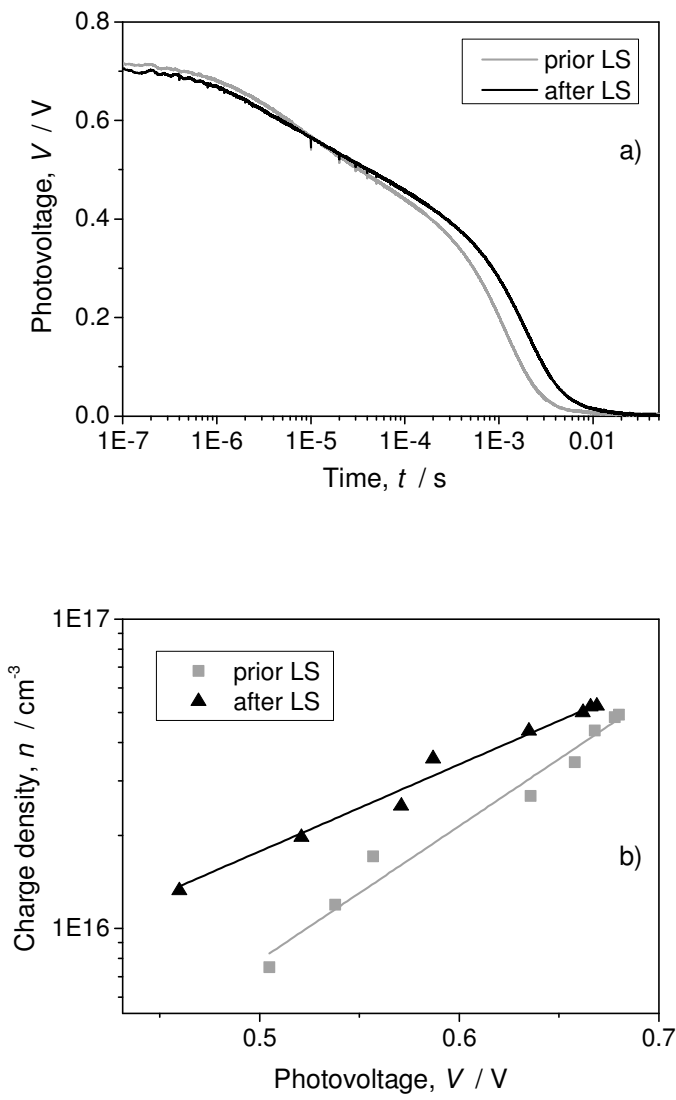

Figure 7. (a) The decay of photovoltage over time before and after light soaking (LS). (b) The dependence of the photovoltage on the charge density of the same device before and after light soaking. 


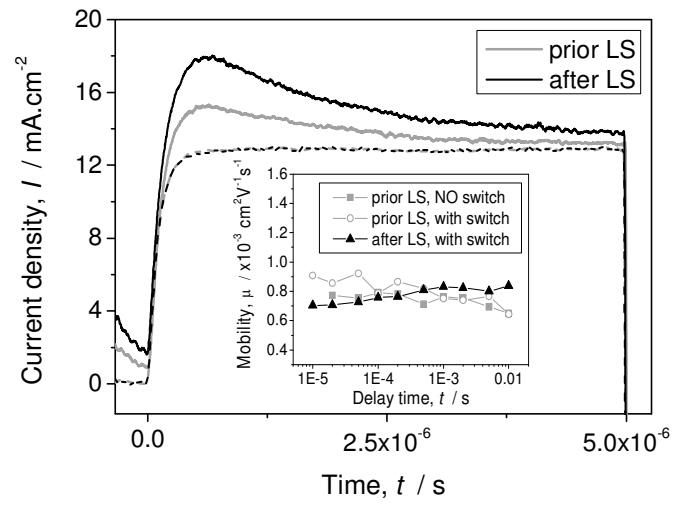

Figure 8. The photo-CELIV curves of the encapsulated KP115:PCBM (1:2) photovoltaic device before and after light soaking (LS), measured using a nanosecond time-resolved switch and a $532 \mathrm{~nm} 0.3 \mu \mathrm{J} . \mathrm{cm}^{-2}$ excitation pulse followed, after a delay time of $100 \mu \mathrm{s}$, by a $4 \mathrm{~V}$ voltage pulse with a width of $5 \mu \mathrm{s}$. The dark CELIV traces are also shown. The inset shows the mobility values over delay times from $10 \mu$ s to $10 \mathrm{~ms}$, prior to light-soaking with and without the switch, and after light soaking with the switch.

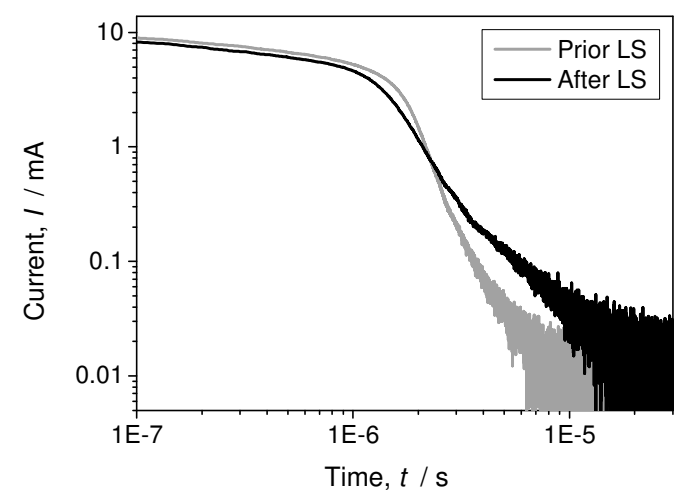

Figure 9. Photocurrent decays of the encapsulated KP115:PCBM (1:2) photovoltaic device before and after light soaking (LS), measured using a $50 \Omega$ resistance, $0.4 \mathrm{~V}$ constant reverse bias and a $1 \mu \mathrm{J} . \mathrm{cm}^{-2} 532 \mathrm{~nm}$ laser pulse. 


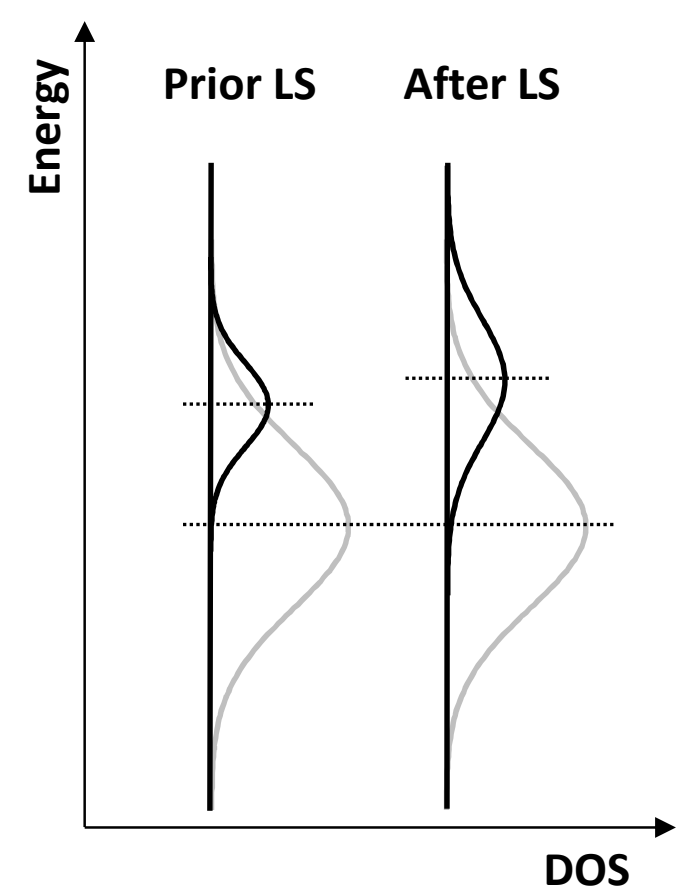

Figure 10. Schematic representation illustrating the effect of light soaking on the density of states of the KP115 HOMO. After light soaking, the bimodal distribution of free (grey curve) and localised (black curve) charge carriers changes, with the distribution of localised states becoming both deeper and broader. The horizontal lines represent the centre of each gaussian.

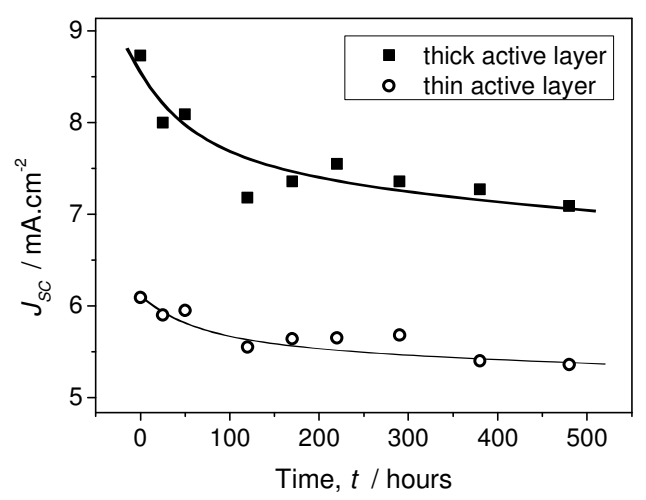

Figure 11. The differences in light soaking behaviour between devices with thick $(\sim 150 \mathrm{~nm})$ and thin $(\sim 70 \mathrm{~nm})$ active layers. 
Photodegradation in encapsulated silole-based polymer:PCBM photovoltaic blends investigated using transient absorption spectroscopy and charge extraction measurements

Tracey M. Clarke,* Christoph Lungenschmied, Jeff Peet, Nicolas Drolet, Kenji Sunahara, Akihiro Furube, and Attila J. Mozer*

Photodegradation of photovoltaic devices based on the novel silole-based polymer KP115 with PCBM has been investigated. The devices show a burn-in phase which was probed using both transient absorption spectroscopy and charge extraction measurements. The decreased photovoltaic performance is attributed to an increased influence from trap states, decreasing the efficiency of charge extraction.

Keywords: charge transport, conducting polymer, organic solar cell, photodegradation, transient absorption spectroscopy

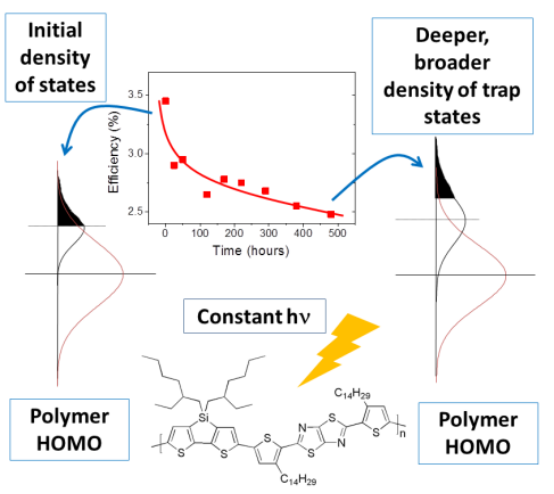


Copyright WILEY-VCH Verlag GmbH \& Co. KGaA, 69469 Weinheim, Germany, 2012.

\section{Supporting Information}

for Adv. Energy Mater., DOI: 10.1002/aenm.((please add manuscript number))

Photodegradation in encapsulated silole-based polymer:PCBM solar cells investigated using transient absorption spectroscopy and charge extraction measurements

Tracey M. Clarke,* Christoph Lungenschmied, Jeff Peet, Nicolas Drolet, Kenji Sunahara, Akihiro Furube, and Attila J. Mozer**

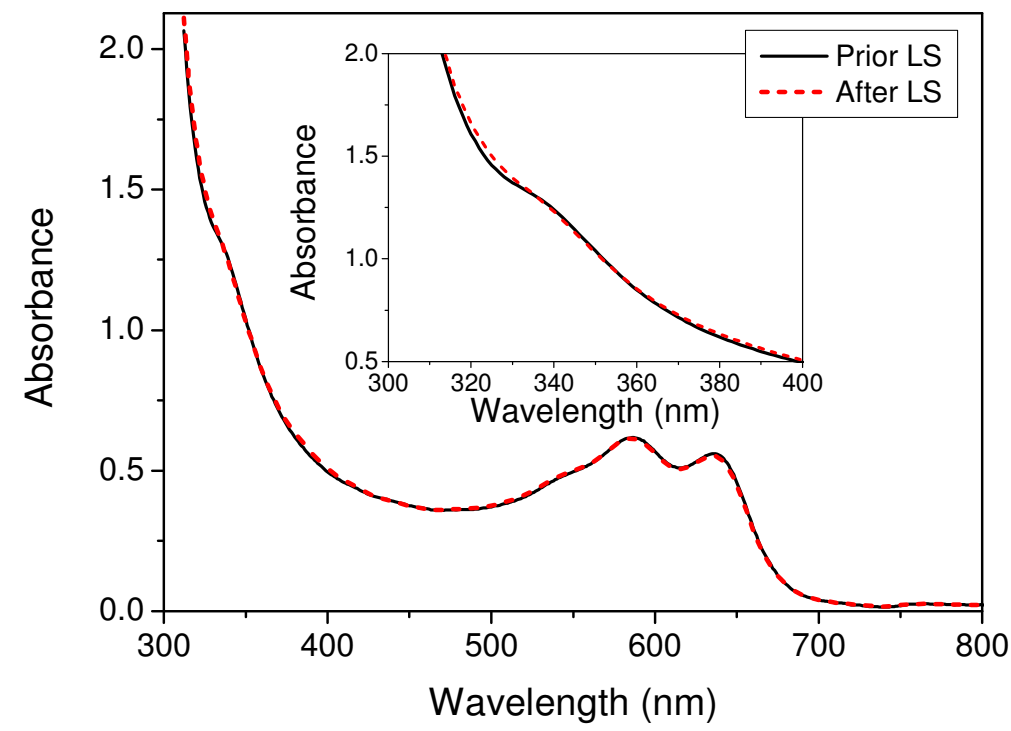

Figure S1. The ground state absorbance spectrum of the encapsulated KP115:PCBM $(1: 2)$ photovoltaic blend film before and after 72 hours of light soaking (LS), with the inset highlighting the PCBM area of the spectrum. 


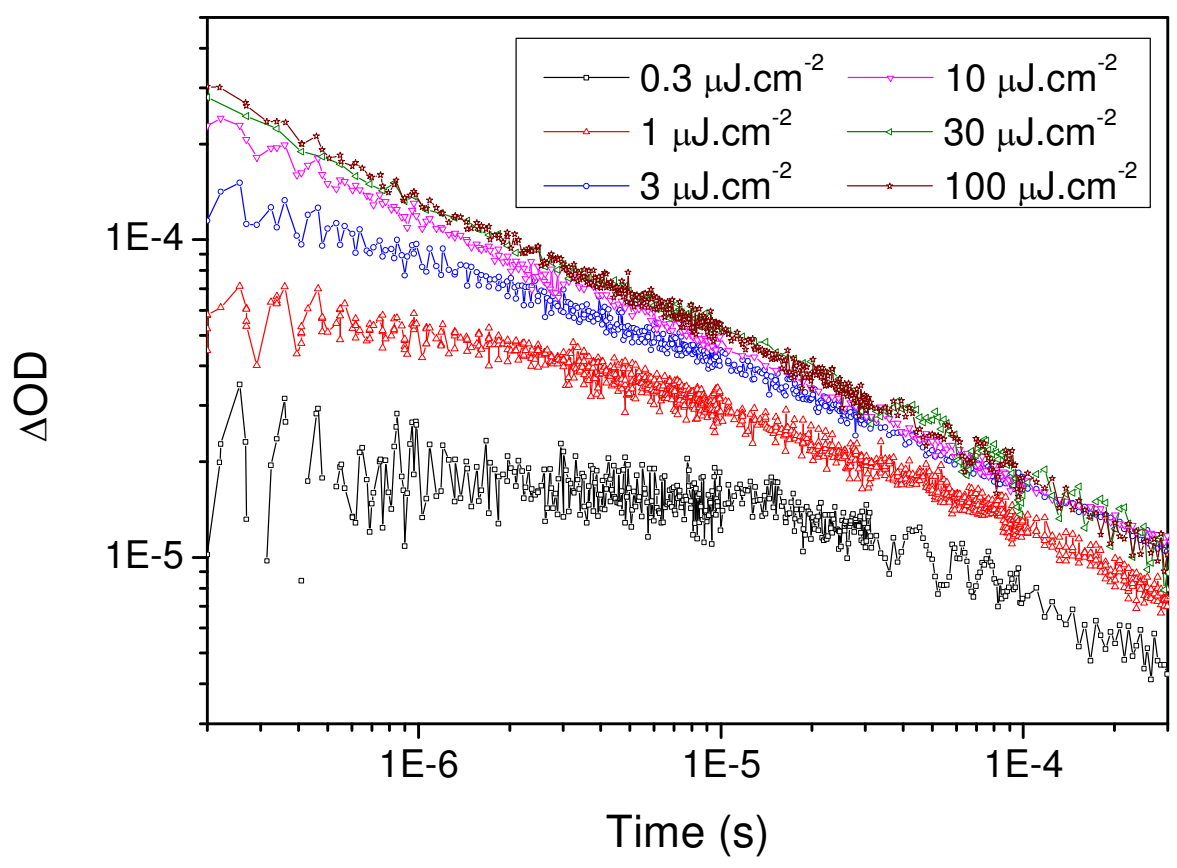

Figure S2. The transient absorption decays as a function of excitation density of the KP115:PCBM (1:2) photovoltaic device before light soaking, using $532 \mathrm{~nm}$ excitation with a probe wavelength of $1000 \mathrm{~nm}$.

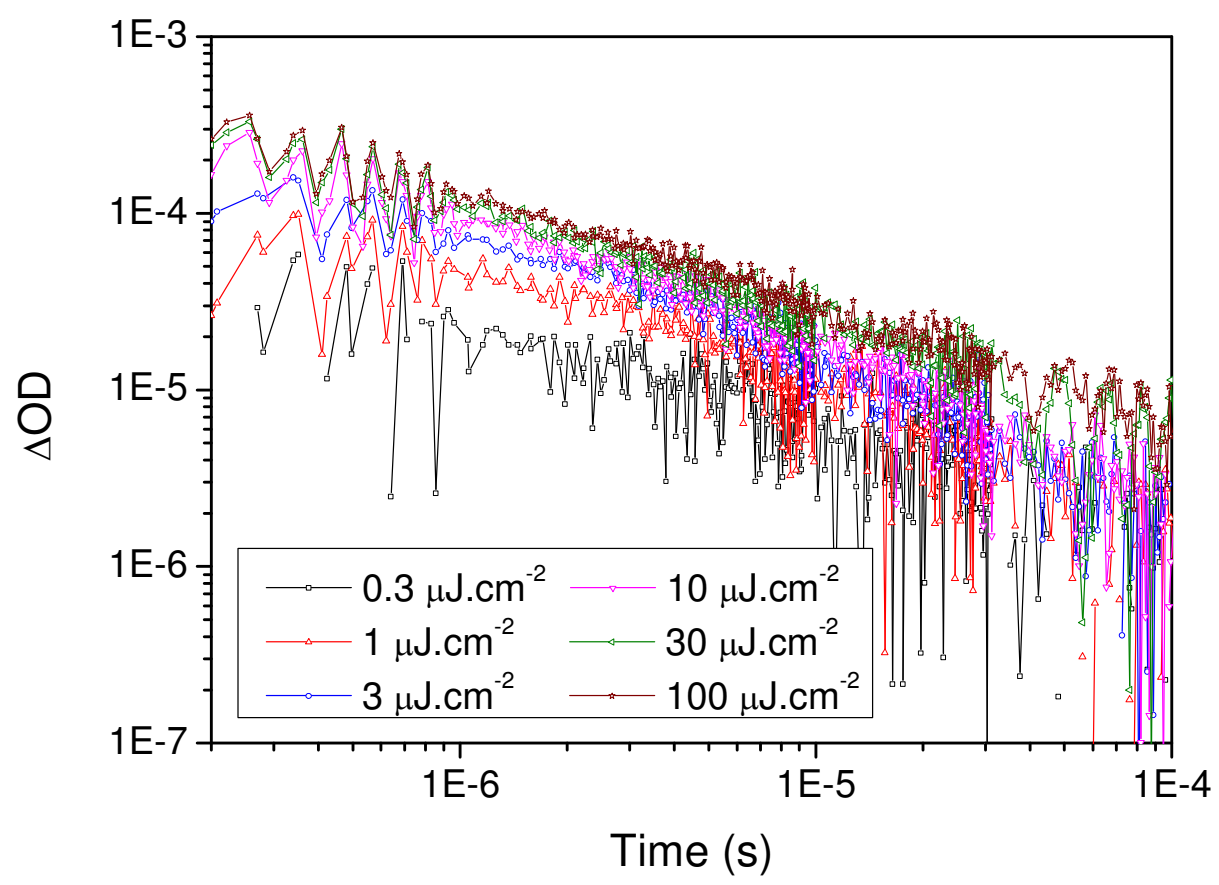

Figure S3. The transient absorption decays as a function of excitation density of the KP115:PCBM (1:2) photovoltaic device before light soaking, using $532 \mathrm{~nm}$ excitation with a probe wavelength of $720 \mathrm{~nm}$. 


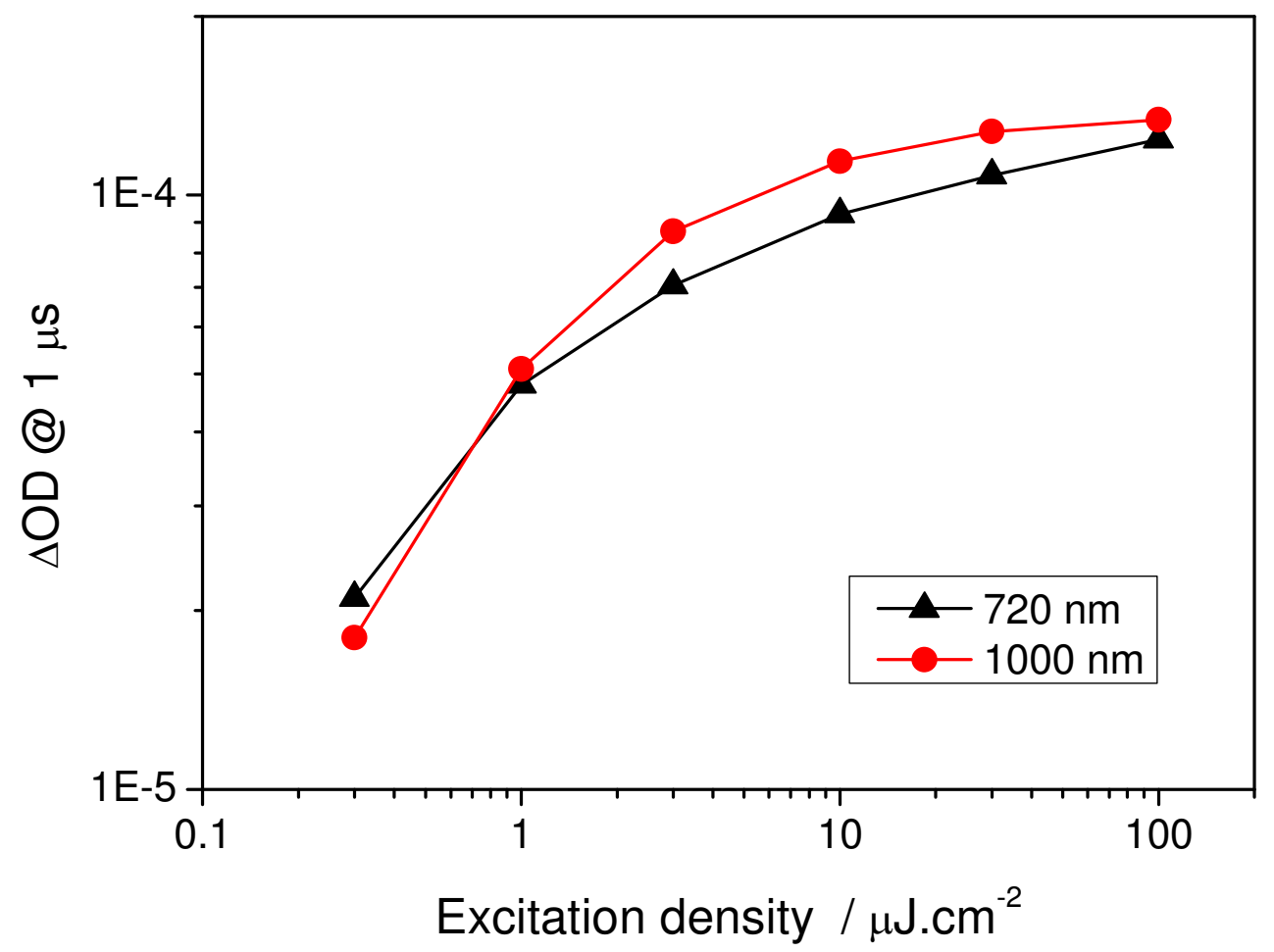

Figure S4. The excitation density dependence of the $\Delta \mathrm{OD}$ at $1 \mu$ s for both probe wavelengths of $720 \mathrm{~nm}$ and $1000 \mathrm{~nm}$, prior to light soaking of the KP115:PCBM (1:2) photovoltaic device.

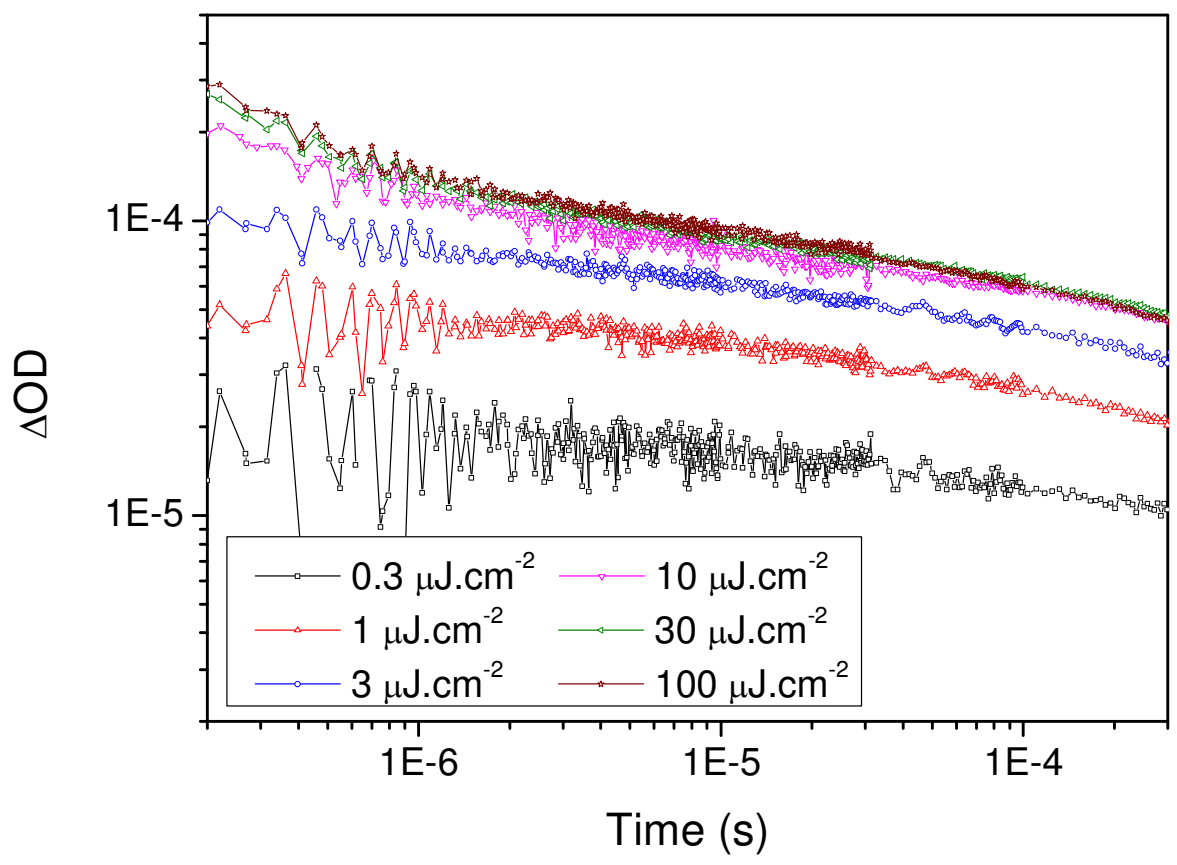

Figure S5. The transient absorption decays as a function of excitation density of the KP115:PCBM (1:2) photovoltaic device after light soaking, using $532 \mathrm{~nm}$ excitation with a probe wavelength of $1000 \mathrm{~nm}$. 


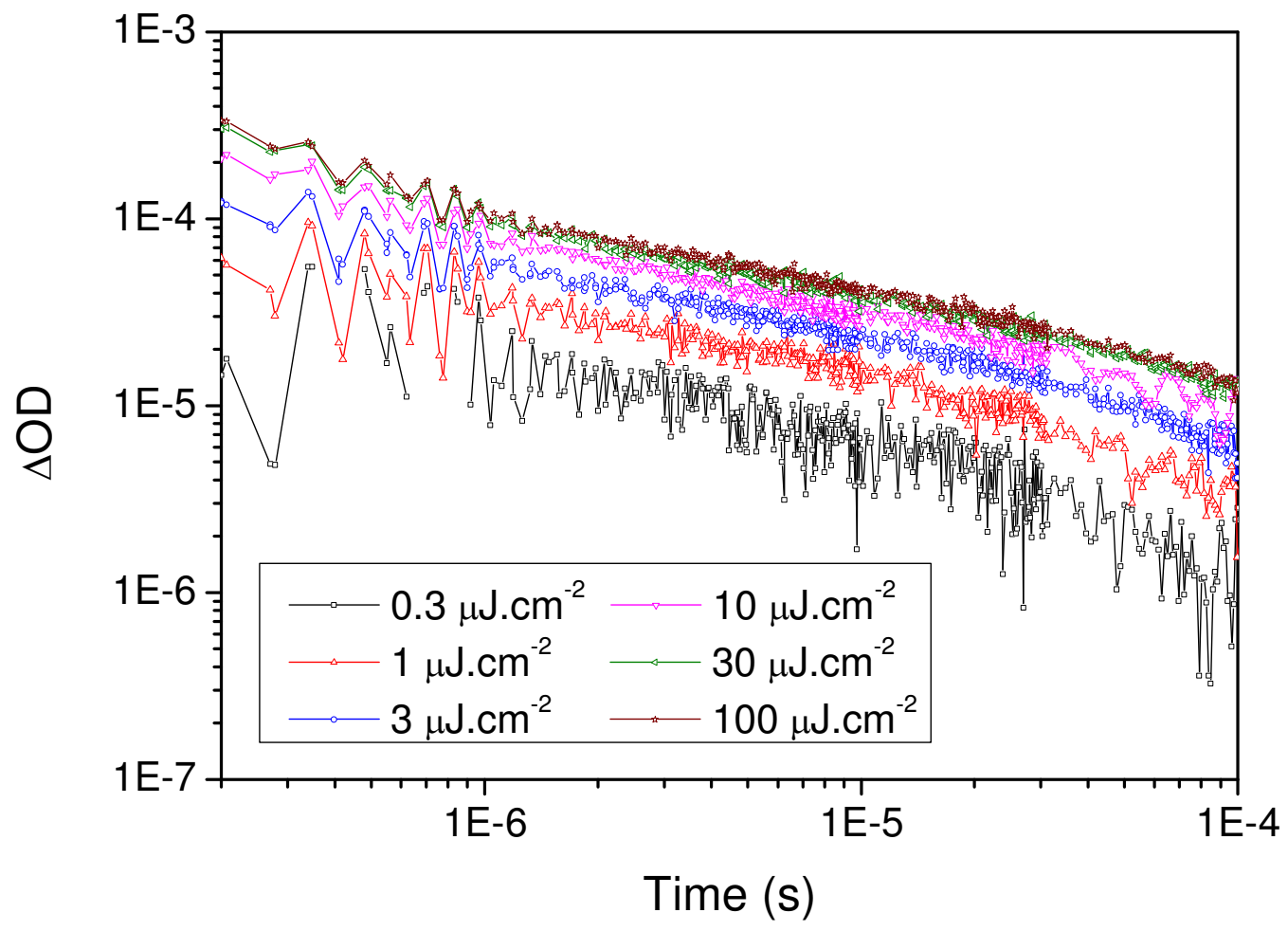

Figure S6. The transient absorption decays as a function of excitation density of the KP115:PCBM (1:2) photovoltaic device after light soaking, using $532 \mathrm{~nm}$ excitation with a probe wavelength of $720 \mathrm{~nm}$. 